\title{
Does a Low-Entropy Constraint Prevent Us from Influencing the Past?
}

\author{
Mathias Frisch \\ University of Maryland, College Park
}

\section{Introduction $^{1}$}

It is part of our common sense conception of the world that what happens now can make a difference to the future but not to the past; events in the present, we believe, can causally influence the occurrence of future events but not of past events. What is the relation between this asymmetry and other physical asymmetries? Is the causal asymmetry fundamental or can our asymmetric notion of cause be shown to be reducible to some other physical asymmetry? There is a venerable tradition in the foundations of physics and the philosophy of science according to which the causal asymmetry is intimately related to the temporal asymmetry embodied in the second law of thermodynamics. This view has recently been forcefully defended by David Albert in (Albert 2000) and by Barry Loewer (Loewer 2007), who argue that the causal asymmetry can ultimately be grounded in the very same facts that give rise to the second law of thermodynamics, chiefly among them a low-entropy constraint on the initial state of the universe.

In this paper I will critically examine aspects of their accounts and will argue that neither account is successful as developed so far. ${ }^{2}$ In section 2 I will briefly summarize the Boltzmannian account of the thermodynamic asymmetry, from which Albert and Loewer aim to

\footnotetext{
${ }^{1}$ I would like to thank the audiences at the 'Time, Chance, and Reduction' workshop in Munich and at the 2007 Shapiro Conference at Brown University, and especially David Albert, Doug Kutach, Barry Loewer, Huw Price, Brad Weslake, and Jim Woodward for very helpful comments and criticisms on earlier versions of this paper.

${ }^{2}$ For discussions of how a third asymmetry - the asymmetry of radiation - may be related to the asymmetries of thermodynamics and of causation, see (Frisch 2000, 2005, 2006b).
} 
derive asymmetries of causal influence and control. Both accounts centrally involve the claim that it follows from the Boltzmannian account that possible macro-evolutions are much more restricted toward the past than toward the future. The statistical mechanical account, as Loewer puts it, results in a time-asymmetric "tree-structure" for possible macro-evolutions. I will criticize this claim in section 3.

While there is a considerable amount of agreement between the two accounts, Albert and Loewer emphasize different routes by which the thermodynamic asymmetry is meant to ground our time-asymmetric notions of causal influence or control. Albert, in the first instance, focuses on a purely physical asymmetry that he takes to be exhibited by suitably small and localized macro-events of the kind that figure in paradigmatic causal judgments (such as that the collision between two billiard balls caused one ball to roll into the corner pocket). In Albert's terminology, such macro-events can provide us with causal handles on the future, but not the past. Loewer's primary focus, by contrast, is on human actions and a putative asymmetry characterizing decision counterfactuals, whose antecedents make reference to possible decisions. In sections 4 and 5 I will raise worries about both of these routes of trying to connect up the statistical mechanical asymmetry to the asymmetry of control or influence.

Albert's and Loewer's aim is to offer an account of how it is that we possess a timeasymmetric concept of causal influence or control by showing that such a concept tracks certain non-causal features of the world given by fundamental physics. Common sense causal claims are by and large concerned with relatively small, 'human-sized' macro events of the kind that could be the result of human interventions. Arguably, then, any account of how we come to possess an asymmetric concept of cause need only be able to reproduce the asymmetry as far as 
causal claims within this domain concerned. ${ }^{3}$ It is, however, part of our notion of causal relations among 'human-sized' macro-events that the temporal asymmetry of causation is strict in the sense that in all paradigmatic or standard circumstances the relation of causation is future directed and that in such cases there is absolutely no backward causation. We believe that our interventions can have an effect on the future development of the world and we also believe that our interventions can have absolutely no effect on the past. A successful entropy account would have to be able to account for this feature of our concept. This does not mean that it has to be a consequence of the account that there is no backward causal influence. Since entropy accounts ultimately appeal to certain probabilistic relations that they take to be derivable from statistical mechanics, they may have the consequence that the causal asymmetry is not strict. Nevertheless, the account has to be able to explain why we take the asymmetry to be strict. ${ }^{4}$ Thus, similar to derivations of the Second Law from statistical consideration it would have to be shown that in paradigmatically causal contexts exceptions to the asymmetry are extremely rare and improbable. It would be a problem for an entropy account, if it implied that there will be widespread backward causal influence in the kind of circumstances that we take to be paradigmatically forward causal.

\section{The micro-statistical account}

\footnotetext{
${ }^{3}$ Thus, Albert rightly argues that the fact that a universe in the shape of Bozo the clown would have to have had a very different past from ours would not undermine his entropy-account of causation (see Albert 2000, 130, fn. 21). Even if Albert's account had the consequence that there is backward causal dependence in this case, this will pose no problem for his account, since this is not the kind of case that could have played a role in our acquisition of causal concepts.

${ }^{4}$ I think it may even be compatible with our common sense notion of causation, that there could be arcane physical circumstances in which there is backward causation. The point I am making here is that we take the asymmetry to be strict as far as common sense, 'billiard ball-like' circumstances are concerned.
} 
According to the Boltzmannian account defended in (Albert 2000), the thermodynamic asymmetry that the entropy of a closed macroscopic system never decreases can be explained by appealing to a time-symmetric micro-dynamics and an asymmetric constraint on initial conditions. If we assume an equiprobability distribution of micro-states compatible with a given macro state of non-maximal entropy, then it can be made plausible that, intuitively, 'most' micro states will evolve into states corresponding to macro states of higher entropy. However, if the micro-dynamics governing the system is time-symmetric, then the same kind of considerations also appear to show that, with overwhelming probability, the system evolved from a state of higher entropy. This undesirable retrodiction, which is at the core of the reversibility objection, can be blocked, if we conditionalize the distribution of micro-states not on the present macrostate but on a low-entropy initial state of the system. Since the reversibility objection can be raised for any time in the past as well, Albert and others argue that we are ultimately led to postulate an extremely low-entropy state at or near the beginning of the universe. Albert calls this postulate "the past hypothesis" $(P H)$.

Positing an equiprobability distribution at some initial time, however, seems to lead to the following problem. If we postulate a uniform probability distribution over the initial state of a system, then the distribution will not be uniform over micro-states compatible with the actual macro-state at later times. If later macro-states have higher entropy, they will correspond to regions of phase space that are vastly larger than the region corresponding to the low-entropy initial state. But, according to Liouville's theorem, regions of phase space evolve into regions of equal size. Thus, positing an equi-probability distribution at the initial time precludes that the distribution is uniform over macro-states at later times and, hence, might seem to preclude us 
from bringing to bear the very considerations that seemed to ensure that entropy is overwhelmingly likely to increase in the first place.

This problem can be solved, if we assume that the phase space region corresponding to the initial macro-state dynamically evolves into a highly fibrillated region such that the microstates that have evolved from the initial macro-state eventually are homogenously distributed over all measurable sub-regions of the system's available phase space. A formal condition that ensures that this assumption is the condition that a system is mixing (see, e.g., Uffink 2006). A dynamical system is a tuple $<\Gamma, \boldsymbol{A}, \mu, T>$, where $\Gamma$ is the system's phase space, $\boldsymbol{A}$ is the set of measurable subsets of $\Gamma, \mu$ is a probability measure, and $T$ is a one parameter group of transformations $T_{t}$ that represents the evolution operators. A dynamical system is mixing, just in case, for all $A, B \in \boldsymbol{A}$

$$
\lim _{t \rightarrow \infty} \mu\left(T_{t} A \cap B\right)=\mu(A) \mu(B)
$$

For such a system, the micro-state at $t$ will with overwhelming probability be 'typical' of the micro-states compatible with the macro-state at $t$, in the sense required for the Boltzmannian account.

Thus, the assumptions of the statistical mechanical account (SM) from which the thermodynamic asymmetry is derived are the following:

(i) time-symmetric, deterministic dynamical micro laws.

(ii) the past hypothesis $\mathrm{PH}$, which characterizes the initial macro state of the universe as a low-entropy condition satisfying certain further symmetry conditions.

(iii) a probability postulate $P R O B$, which postulates a uniform probability distribution over the physically possible initial micro-states of the universe, compatible with the past hypothesis $P H$. 
(iv) an assumption of mixing or dynamic instability of possible micro-evolutions. This account of the entropy-asymmetry has been challenged (e.g., by Winsberg 2004; Earman 2006), but I do not want to discuss these criticisms here. In what follows I will assume that the account can successfully explain the thermodynamic asymmetry and ask whether it can be extended to explain an asymmetry of influence as well.

\section{Trees or webs}

Immediately after his own summary of how the micro-statistical account appealing to $P H$ and $P R O B$ accounts for the entropy asymmetry, Loewer presents the following figure (figure 1), which, he says, "is a depiction of possible evolutions of micro and macro states that should provide an idea of how all this goes." (Loewer 2007, 300) [[insert figure 1 roughly here]]

In this figure thin lines represent possible micro histories of the universe and thick cylinders represent possible macro histories. Possible macro-histories, Loewer claims, exhibit a tree structure: even though the evolution of micro-histories is assumed to be deterministic, the evolution of macro-histories is future-indeterministic in that more than one future macro-history will in general be compatible with the macro-state of the world at a time. Indeed, there are different possible macro-futures that get assigned relatively high probabilities conditional on the present macro state. This contrasts with the probabilities assigned to different past evolutions:

From a typical macro state in the middle of the actual macro history there will be branching in both temporal directions but there will be much more branching where the branches have substantial probability in the direction away from the time of the $P H$ than back towards it. The overall structure is due to the fact that 
the macro state at $t$ (in the middle) must end up in the direction of the boundary condition at which $P H$ obtains (the direction we call 'the past') satisfying $P H$. (Loewer 2007, 302)

Loewer reiterates the same point further down:

$[P H$ and $P R O B]$ determine an objective probability distribution over all nomologically possible micro histories (and a fortiori over all macro histories and all macro propositions). Even though the underlying micro dynamics is deterministic macro histories form a tree structure branching towards the future (away from the time at which $P H$ holds). (Loewer 2007, 307)

That is, the objective probability distribution determined by $P H$ and $P R O B$ forms a branching tree structure - a tree structure that is "due to the fact" that $P H$ provides a constraint on possible evolutions.

We can express this structure somewhat more formally by introducing the notion of quasi-determinism:

(QD) A system is quasi-deterministic at $t$ relative to some time $t^{\prime}$ and some set of mutually exclusive macro-states $\boldsymbol{M}$, exactly if there is a state $M_{i}$ in $\boldsymbol{M}$ such that $\mathrm{P}\left(M_{i}\left(t^{\prime}\right) / S(t)\right)$ is close to 1 , where $S$ is the state of the system at $t$.

The probabilities here (and throughout this paper) are the ones induced by the statistical mechanical probability distribution and conditionalization on the dynamical micro-laws and the past-hypothesis $P H$ is left implicit. The claim that the universe exhibits an asymmetric treestructure is equivalent to the conjunction of the following two claims: ${ }^{5}$

\footnotetext{
${ }^{5}$ Since Loewer only claims that there is "much more branching" toward the future, one might think that conditions (1) and (2) are stronger than what Loewer would want to endorse. We do not need to settle this question in general here. The important issue will be whether there is
} 
(1) The world is not quasi-future-deterministic; or more precisely: For all times $t$ there is a $\Delta t$, such that for all times $t^{\prime}>t+\Delta t$ and all $M$, the world is not quasi-deterministic at $t$ relative to $t^{\prime}$.

(2) The world is quasi-past-deterministic at all times $t$ with respect to all times $t^{\prime}, t^{\prime}<t$, and all M.

I have shown in (Frisch 2006a) that from a formalization of the tree-structure similar to QD one can indeed derive the asymmetry of decision counterfactuals that is at the core of Loewer's account. I have also suggested, however, that the claim that the SM account implies a tree-structure of possible macro-evolutions is problematic and here I want to develop this latter claim in more detail.

Since macro-states closer to equilibrium occupy vastly larger regions of phase space than states further away from equilibrium, it follows from Liouville's theorem that there will be many possible different non-equilibrium states far from equilibrium that evolve into the same state closer to equilibrium in the future. This suggests that there may be many more changes to the micro-state of a system close to equilibrium associated with different macro-pasts further away from equilibrium than there are changes to the micro-state of a system far from equilibrium associated with different macro-futures closer to equilibrium. Merely comparing the phasespace volumes associated with macro-states at different times suggests that possible macroevolutions may exhibit an upside-down tree structure. ${ }^{6}$

widespread branching toward the past involving the kinds of event that feature in paradigmatically causal judgments.

${ }^{6}$ Since Loewer represents possible macro-evolutions in his diagram of a possible tree structure (figure 1) by cylinders of constant diameter, the diameter cannot be taken to represent phase space volumes. If we wanted to include a representations of the phase space volumes associated with macro states in the diagram, possible macro-evolutions would have to represented by cones of dramatically increasing widths towards the future. As a rhetorical device, Loewer's diagram 
We can distinguish two worries here: first, focusing on the future 'end' of the treestructure, is it indeed a consequence of the SM account that there will be no significant reconvergence of branches, and that there are no times with respect to which thermodynamic systems are quasi-deterministic? And, second, focusing on the past 'end,' is it a consequence of the account that the past is quasi-deterministic at all times with respect to the initial time $t_{P H}$ at which $P H$ holds?

That future evolutions are not quasi-deterministic might seem to follow from the assumption of mixing. If a system is mixing the conditional probability $P\left(M(t) / M_{0}\right)$ of a macrostate $M(t)$ given the initial state $M_{0}$ depends only on the phase-space volume associated with $M(t)$ and is independent of $M_{0}$. Yet the mixing assumption alone does not imply the failure of quasideterminism for all future times. If there is a single equilibrium macro-state $M_{e}$ that takes up the overwhelming majority of the phase space region available to a system, then $P\left(M_{e}\right)$ can be close to one and the system is quasi-future-deterministic with respect to all times after which the system reaches equilibrium.

This point holds for thermodynamic systems of all sizes - to the extent that the Boltzmannian account applies to these systems - ranging from small macroscopic quasi-isolated systems to the universe as a whole. Consider, for example, the paradigmatic thermodynamic system - a body of gas that is initially confined to the right half of a container and, after a partition is removed, spreads out until it is distributed evenly throughout the container. Since most of the phase space accessible to the gas is associated with its equilibrium state, the SM account allows us to predict that the gas will be overwhelmingly likely to end up in that state-

lends far more plausibility to the thesis of macro-branching toward the future, than a picture of cones that branch at the same time as they dramatically increase in width. (When you try to draw this, you'll quickly run out of space into which branching could occur.) 
the gas evolves quasi-deterministically with respect to the final equilibrium state. At the other extreme, current cosmology suggests that the universe as a whole, too, may be quasideterministic with respect to its future equilibrium state, in which ionized stable particles, i.e. protons, neutrons, and electrons, are distributed evenly throughout the cosmos at a density approaching zero (see e.g. Baez 2004).

While Loewer is obviously right in suggesting that there are many systems that are open to the future - there clearly is widespread macro-branching toward the future - thermodynamic considerations imply that there also is widespread reconvergence of possible macro-histories. Thus, at the cosmological level, even though the initial state of the universe might not determine the large-scale distribution of matter before elementary particles begin to 'boil off' in the final evolution towards equilibrium, different cosmological macro-histories will converge toward the final equilibrium state. Similarly, there is convergence at the level of 'human-sized' macrosystems: no matter which part of the container a body of gas occupies initially, after the partition is removed the gas will spread until it is uniformly distributed throughout the container.

As a simple case exhibiting both branching and reconvergence consider the example Albert uses to motivate postulating a past-hypothesis and the existence of macro-branching (Albert 2000, 82ff). Albert imagines a system consisting of ice cubes that drop into glasses of water after sliding down a device similar to a Galton board. The same low-entropy initial state, with the ice cubes collected at the top, will indeterministically evolve into different macro-states given by different distributions of ice cubes in the glasses at the bottom of the board. Yet if we imagine that the ice cubes have several macroscopically distinct shapes of the same volumes, then there will be macroscopically distinct distributions of ice cubes in the glasses that will eventually evolve into the same macro-states once the ice is fully melted. And if we further 
assume that at the end of our experiment all glasses of water are emptied into a single bucket, all possible macro-histories that diverge after the ice is released a the top of the Galton board will reconverge - no matter what the shapes or volumes of the ice cubes are and no matter what path they take down the board. That is, the final state of the system when the all the water is collected in a bucket is not quasi-deterministic with respect to past times when the ice cubes were distributed among the different glasses, even if we impose as additional constraint that all macrohistories are constrained to have originated in the state where the ice cubes were collected in a container at the top of the Galton board.

Now, there are discussions in the literature on counterfactuals that suggest that there is a crucial consideration that has been missing from our examination so far-the role of records or traces of the past. These discussions often invoke Kit Fine's famous example of Nixon's pushing the button that leads to a nuclear holocaust. It is often suggested that the many traces Nixon's action (or inaction) leave in the world play an important role in making convergence of 'button-pushing worlds' with 'non-button-pushing worlds' difficult. In the case of the ice cubes sliding down the Galton board drops of water on the board or my memories of observing a particular ice cube slide down a certain path might constitute such traces.

But it is easy to exaggerate how frequent and persistent macro-traces are. In fact, it is precisely the thermodynamic behavior of systems that often either prevents the formation of macro-traces or leads to the disappearance of such traces. Whatever else the connection between $\mathrm{PH}$ and the existence of records is, one central role played by the thermodynamic arrow is as a destroyer of macro-records and macro-traces. Thus, any drops of water left on the Galton board will eventually evaporate; and since what path a particular ice cube took does not have the same momentous consequences for the fate of the Earth as Nixon's decision whether to push the 
button, I will soon forget any details of what I observed (and may well forget altogether that I ever conducted the experiment). Moreover, there will not be any other macroscopic 'traces' of the experiment. While light waves will be reflected differently by the ice cubes depending on their path, due to the multiple scatterings of photons off the laboratory walls and the air molecules, these differences will leave no macroscopic traces by the next day. We might even imagine that there are different lamps that light up depending on what path an ice cube slides down. By the next day — and in fact much sooner - there will be no macroscopic traces of a particular lamp's having been on when ice cube 17 slid down the board. Due to the thermodynamic behavior of the walls of the laboratory and of the atmosphere, the macro-state of the world tomorrow will be independent of what the outcome of my experiment is today.

Examples similar to this one can be multiplied indefinitely. While there indubitably are many systems which for some finite time do not evolve quasi-deterministically, there are also many cases like the ones I just described — cases for which differences even in the current macrostate will eventually 'wash out,' for which the system's macro-history throughout some period $T$ will leave no macroscopic traces in the future, and for which different macro-states will evolve quasi-deterministically into one and the same future macro-state.

I have argued that the assumption of mixing is not enough to ensure that a system is future quasi-indeterministic with respect to its equilibrium state and that it is a consequence of the thermodynamic behavior of systems that there will be reconvergence of possible macrohistories even for systems that do not evolve quasi-deterministically during some time-interval $T$. Can mixing at least ensure that the evolution toward equilibrium is not deterministic? It is far from clear that the answer is 'yes.' First, all we can conclude from the assumption that a system is mixing is that after a sufficiently long time the probability of finding a system in a given 
macro-state is proportional to the phase-space volume associated with that state. That is, we can conclude from the fact that a system is mixing that it will end up in an equilibrium state, but we cannot draw any inferences at all about how it will get there.

Second, as (Earman 2006) has argued, if we were able to show that all thermodynamic macroscopic systems had different possible macro-futures that receive substantial probability, we might be showing too much, as it were, and our theory would be empirically inadequate. While there clearly are systems that are dynamically unstable on the macro-level, there also are many systems that do not exhibit any macroscopic instability and are quasi-future-deterministic. Indeed, many paradigm cases of causal or time-asymmetric counterfactual judgments concern such quasi-deterministic macro-systems. Not only might we want to endorse the claim that had the proverbial butterfly not flapped its wings there would not have been a storm - an example of a causal counterfactual concerning a dynamically unstable system—but we might also want to say that had I not stepped on the brake my car would not have come to a halt at the red light — an example of a causal counterfactual concerning, hopefully, a quasi-deterministic system. One might worry, then, how we can recover the apparently deterministic macro-evolutions of many systems from the assumption of dynamic instability on the micro-level.

The picture that has emerged is not one of an asymmetrically branching tree structure, but rather that of a web of possible macro-histories that branch and reconverge. Whether at its future end the web of possible macro histories for the universe converges into a single strand, is a question for cosmology to decide. But the sub-web characterizing the history of Earth and many of the even lower-dimensional 'sub-webs' characterizing human-scale subsystems on Earth involve a large amount of convergence of strands, as well as branchings. Moreover, there are 
many small macro-systems that over (humanly) significant stretches of time evolve quasideterministically and do not exhibit any branching at all.

So far I have focused on Loewer's claim that there is branching toward the future without widespread reconvergence. I now want to turn to his claim that it follows from the SM-account that the macro-evolution of the universe is quasi-past-deterministic with respect to an initial time $t_{P H}$. Above I expressed the past-hypothesis as the constraint that the initial macro-state of the universe was $a$ low-entropy state satisfying certain further symmetry conditions. But if this indeed is what the past-hypothesis says, an additional problem arises for Loewer's claim that the past is closed: It does not seem to follow from the constraint that micro-histories originated in $a$ very low-entropy state that the macro-past is the unique actual low entropy past. That is, counterfactual micro-histories may have originated in low-entropy macro-states distinct from the actual low entropy past. Consider once more a system consisting of a gas in a box and assume that the gas could have started out in one of two possible low-entropy initial states, confined either to the right or the left half of the container. Let us assume that in the actual world the gas started out in the left half of the container and then spread out until it reached equilibrium, filling the entire container. Then, according to the reversibility objection, most changes to the final micro-state will be associated with a high-entropy past, since most micro-states compatible with the final equilibrium state will have evolved from equilibrium initial states. What if we assume a 'past-hypothesis' and constrain changes to the final micro state to those that evolved from $a$ low entropy initial state? The phase space regions corresponding to the two initial states - the gas confined to the right or to the left half of the box-will evolve into highly fibrillated regions. If we assume that the system is mixing, each coarse-grained 'box' of phase space will have the same proportion of points that have evolved from the two initial regions. That is, intuitively, 
while the overwhelming majority of points in each box of phase space lie on trajectories that have evolved from high-entropy pasts, the same number of points in each box lies on trajectories that originated in the two low-entropy states. Given the final macro state, the system is as likely to have evolved from the non actual low-entropy past where the gas would have been confined to the right half of the container as from the actual past and adding a low-entropy constraint in the past does nothing to privilege the actual low-entropy past.

If $\mathrm{PH}$ merely restricts macro-histories to have originated in some (suitably symmetric) low-entropy state, then Loewer's conclusion that the universe is quasi-deterministic with respect to $t_{P H}$ seems unwarranted. But Loewer himself characterizes $P H$ differently: He says that $P H$ is "a statement specifying the macro state of the universe at one boundary." (Loewer 2007, 300, my italics). That is, according to Loewer's reading, the past-hypothesis restricts possible microhistories to have originated in the actual low-entropy past state and this restriction trivially ensures that all possible macro-histories originated in one and the same macro-state. But can we assume the actual initial macro-state as constraint, without begging the question, in an account that is meant to derive a temporal asymmetry of counterfactuals?

Loewer's explicit aim is to provide a broadly Lewisian account of a counterfactual asymmetry and he contrasts his and Lewis's strategy, on the one hand, with Jonathan Bennett's, on the other, who does not offer an explanation of the asymmetry but simply assumes that counterfactuals are evaluated by keeping the past fixed. Loewer says:

I think that Bennett's account does a pretty good job of characterizing a conditional that matches core uses of the counterfactuals that interest us. [...] However, Bennett's procedure for evaluating counterfactuals assumes the 
distinction between past and future (since forks are to the future) and so it does not provide a scientific explanation of time's arrows. (Loewer 2007, 309-310)

Thus, in order to provide a scientific explanation of the asymmetry, we cannot merely assume the asymmetry by holding the past fixed and allowing only the future to vary, but have to derive this asymmetry from the global distribution of matters of fact in the actual world in conjunction with the laws.

One might worry, then, that by the very fact that Loewer assumes $P H$ as a timeasymmetric constraint he, like Bennett, is putting in the asymmetry by hand. Both Bennett and Loewer, it seems, stipulate a time-asymmetric constraint on how past states of the world may vary, and from this derive that counterfactuals are time-asymmetric. To be sure, Bennett's constraint goes beyond Loewer's - he stipulates that we hold fixed the entire macro-history in one temporal direction, while Loewer only fixes the macro-state at the past temporal end - but Loewer's constraint may strike one as similarly question-begging, if our goal is to provide a scientific explanation of a temporal asymmetry of counterfactuals. The only "scientific contribution" to Loewer's account might be that the dynamical laws need to ensure that counterfactual past micro-evolutions converge quickly enough with the actual macro-past.

Loewer's reply to this worry is that the initial macro-state of the universe plays a special role in our overall scientific conception of the world. Loewer himself tries to capture this role by proposing a broadly Lewisian account of laws and suggesting that the actual initial macro-state is part of the Lewisian Best System. Yet he apparently also believes that the special scientific status of the initial macro-state can be motivated independently of Lewis's account of laws. What, then, is the special role played by the $P H$ and does that role provide us with good reasons for assuming the actual initial macro-state (rather than just $a$ low-entropy state) as constraint on 
possible macro-histories?

First, in the Boltzmannian account, $P H$ plays a central role in deriving the thermodynamic asymmetry. Thus, Loewer supports affording $P H$ a special role by saying that it "underwrite[s] many of the asymmetric generalizations of the special sciences especially those in thermodynamics and these generalizations are considered to be laws." (Loewer 2007, 304) But in order to derive the thermodynamic 'laws' it is sufficient to assume that the universe began its life in $a$ low-entropy state (in addition to $P R O B$ ). Thus, the foundations of thermodynamics do not provide us with a reason to accept Loewer's version of $P H$ as constraint instead of the one I proposed.

Second, both Albert and Loewer point to the explanatory role the actual macro-state of the early universe plays in current cosmology. Thus, they maintain that any macro-state that results from a small hypothetical alteration to the actual present macro-state is constrained to have evolved from the actual initial macro-state that cosmology will eventually present to us. But the plausibility of this claim relies on an equivocation on the notion of macro-state. A macro-state is associated with a coarse-graining over the phase-space of a system and in different contexts, different coarse-grainings are appropriate. In the case of the kind of counterfactuals associated with paradigmatically causal claims, the right level of description is one referring to medium-sized, 'human-scale' objects, whose states are characterized in units such as $1 \mathrm{~m}$ or $1 \mathrm{~kg}$. In the context of astronomy or cosmology we are interested in the distribution of stars and galaxies and demanding that macro-states ought be specified to a precision of the location of medium-sized objects would be absurd. Appropriate units in the latter context are, for example, the astronomical unit $1 \mathrm{AU}=1.5 \times 10^{11} \mathrm{~m}$ or the solar mass $1 \mathrm{M}=1.9 \times 10^{30} \mathrm{~kg}$. Thus, even if we grant that a specification of the actual initial macro-state of the universe provides a scientifically 
legitimate and non-question-begging constraint on possible macro-evolutions, the constraint can only be a specification of the cosmological, coarse-grained macro-state. Any specification of the initial macro-state more fine-grained than that does not play a scientifically explanatory role. ${ }^{7}$ But just as there are many micro-states compatible with a given fine-grained macro-state, there are many fine-grained macro-states (specifying, for example, the exact distribution of small rocks on a planet's surface) compatible with a more coarse-grained macro-state.

One might think that the specific nature of the macro-state of the early universe provides a reply to this worry. According to current cosmology, matter was distributed smoothly shortly after the Big Bang. (A smooth matter distribution, it is often argued, represents a state of extremely low gravitational entropy, and hence, as matter clumped to form stars and galaxies, the gravitational entropy of the universe increased.) Thus, one might think that there is just a single initial macro-state tout court — that is, even just a single fine-grained macro-state—-that satisfies the conditions revealed to us by cosmology. While there can be many different macro-states that exhibit the same amount of gravitational clumping, there seems to be only a single macro-state characterized by a completely smooth matter distribution —a state that is smooth at all levels of coarse-graining.

But this reply fails for two reasons. First, its premise is false. The macro-state of the early universe was not completely smooth, even on a cosmological level and-fortunately for contemporary cosmology — exhibited density fluctuations large enough to function as seeds for

\footnotetext{
${ }^{7}$ Within Loewer's preferred Lewisian account of laws this point can be made as follows: Including a description of the universe's fine grained, human-scale initial macro-state in our deductive system will vastly complicate the system without providing us much (if any) gain in informativeness.
} 
the formation of stars and galaxies. ${ }^{8}$ Second, the inference from a distribution that is smooth at one level of coarse-graining to one that is smooth at all levels is not sound. It is part of the Boltzmannian account that the micro-state of the early universe was one that is 'typical' given the known macroscopic constraints. This means that, if the association between a smooth matter distribution and low gravitational entropy is correct, the SM account implies that the early universe is overwhelmingly probable to have exhibited as much gravitational clumping as is compatible with our cosmological evidence.

Thus, neither statistical physics nor cosmology provides us with scientific reasons to take the actual fine-grained or human-scale macro-state as constraint on possible fine-grained macroevolutions. The Boltzmannian account requires as premise only that the universe began its life in $a$ state of extremely low entropy and cosmology restricts that state to one that is characterized by an approximately smooth matter distribution, but with density fluctuations large enough to be compatible with many different fine-grained macro-states.

\section{Causal handles}

In the last section I have argued that it is a consequence of the thermodynamic arrow that there will be convergence among different possible macro-evolutions and that there will be many cases where small differences in the macro-state of a system at one time leave no macro-traces in the system's future. In this section and the next I will show that this result leads to problems both for Albert's of causal handles and for Loewer's account of decision-counterfactuals.

Albert argues that it is a consequence of the Boltzmannian account that the present contains multiple causal handles on the future but (almost) no causal handles on the past. If we

\footnotetext{
${ }^{8}$ The density fluctuations are of the order of 1 part in 100,000. By comparison, differences in mass distribution of interest to us are of the order of $10^{-30}$ times the mass of the sun.
} 
constrain the remote past of any physical system, he maintains, then only very few and special alterations to the present are associated with a different recent past, while many such alterations may lead to different futures. To illustrate this point Albert asks us to consider a collection of idealized billiard balls on a frictionless plane such that ball 5 is currently stationary with the additional constraint that ball 5 was moving 10 seconds ago. Given this additional constraint, the fact that ball 5 has been involved in a collision in the past 10 seconds is nomically determined by facts about the present state of ball 5 alone. That is, alterations to the present state of the balls not involving changes in the state of ball 5 cannot change the fact that ball 5 was involved in a collision during the last 10 seconds. Yet there are many changes to the state of the balls not involving ball 5 that will result in a different future evolution of ball 5. From this Albert concludes that there are a far wider variety of 'what we might call causal handles on the future of the ball in question here, under these circumstances, than there are on its past' (Albert 2000, 128) In this example the constraint that ball 5 was moving is meant to play the role of a 'pasthypothesis' and the current state of ball 5 functions as a record of the past collision. More generally, then, Albert claims that if we postulate $P H$ as constraint on all possible macroevolutions, then this imposes almost no additional restriction on possible future macroevolutions, while it restricts non-actual present macro-states that are the result of small macrochanges to the actual present state to have evolved from the actual macro-past- - that is, it follows from imposing $\mathrm{PH}$ as constraint on all possible macro-histories that there are many more causal handles on the future than on the past.

In Albert's example the current state of ball 5 together with the past constraint nomologically determine that ball 5 was involved in a collision. In the general case, however, $P H$ in conjunction with certain local facts about the current macro-state assigns probabilities 
strictly less than one to the occurrence of past events. Many records or traces of the past do not determine the occurrence of the events of which they are records but only raise the probabilities of their occurrence. Thus, the general definition of a causal handle is as follows: A macro-event $C(t)$ is a causal handle on an event $E\left(t^{\prime}\right)$, just in case the occurrence of $C$ (significantly) affects the probability of $E$. That is, $C(t)$ is a causal handle on $E\left(t^{\prime}\right)$ exactly if $\mathrm{P}(E / C \& M(t)) \neq P(E / M)$. $M(t)$ is the actual macro-state at $t$ outside of the region where $C$ occurs and contains any putative records of $E$ at $t$. On Albert's proposal, we evaluate the results of small hypothetical changes to the present by keeping the present macro-state fixed except for the small change and then determine how this counterfactual macro-state evolves in accord with the constraint given by the SM account — with one important qualification: Albert assumes that, in addition to any macrorecords of an event, we also hold fixed any putative memories we might have of that event, even though memories might be physically realized by micro-states.

Albert's thesis that there are (almost) no causal handles on the past is tantamount to a screening-off condition. $C(t)$ is not a causal handle on some past event $E$ just in case the rest of the macro-state at $t$ screens off $E$ from $C$ - that is, $\mathrm{P}(E / C \& M(t))=P(E / M)$. But for events $E$ that leave at most a small number of traces in the present, this condition can easily fail. Take an event $E\left(t^{\prime}\right)$ that has only two distinct macro-traces $C_{1}$ and $C_{2}$ at some later time $t . C_{1}$ and $C_{2}$, intuitively, are both effects of $E$. Thus, while $E$ as the common cause of $C_{1}$ and $C_{2}$ might screen off $C_{1}$ from $C_{2}$, it will not in general be the case that Albert's condition is satisfied and that one effect screens off the cause from the other effect. Indeed, the presence (or absence) of additional traces of an event — that is of additional evidence for the event's occurrence — can radically alter the probability of that event. Albert's Galton board can again serve as an example. That a particular ice cube landed in the second glass from the left, say, constitutes a trace of it having 
slid down to the left of the first pin. (For a board with $n$ rows of pins where the probability at each pin of the ice cube sliding down on one side is equal to $1 / 2$, this probability is $P($ left/second glass to left $=1-1 / n$.) Now let us imagine that the ice cube can dislodge a little ball as it slides down the board and that where the ball ends up also functions as a probabilistic record of the ice cubes path down the board. It is then easy to set up the probabilities in such a way that both the little ball's present position and the ice cube's landing in the second glass come out as causal handles on the path of the ice cube past the first pin. That is, it is easy to set things up such that (keeping the present condition of the ice cube in the glass fixed) there can be many alterations in the present condition of the little ball which would alter the probabilities about whether or not the ice cube slid down to the left of the first pin. ${ }^{9}$

Again, there is nothing unusual about this example. There are many cases where additional evidence affects the probabilities of past events, and hence, according to Albert's account, would classify as causal handle on the past. It seems to me that to the extent that Albert's thesis may appear intuitively plausible, this rests on at least one of the following two assumptions. First, Albert's thesis is true if we demand that a trace (together with the pasthypothesis) nomologically determines the event's occurrence. But while this assumption might hold in the idealized billiard ball case Albert considers, it clearly is not true in general. Second the thesis would follows, if we assumed that each event leaves sufficiently many and varied traces that each trace taken individually only marginally affects the probability of the event's occurrence. But as I have argued in the last section, it is a consequence of the thermodynamic arrow that this assumption is often false. There are many mundane (and paradigmatically

\footnotetext{
${ }^{9}$ And this is meant as an explicit contrast with what Albert says about the billiard ball case on the top of page 127 in his book.
} 
causally related) events that leave no or only very few traces in the future. ${ }^{10}$

\section{Decision counterfactuals}

By contrast with Albert's account, Loewer's account of an asymmetry of control focuses primarily not on a purely physical asymmetry but on an asymmetry involving agents. Loewer argues that the SM account underwrites an asymmetry of decision counterfactuals. A decision counterfactual is a probabilistic counterfactual of the form 'If at $t$ I were to decide $D$, then the probability of $B$ would be $p^{\prime}$, which is true exactly if $\mathrm{P}(B / M(t) \& D(t))=p . M(t)$ is the complete macro-state at $t$ and the decision $D(t)$ is an event "smaller than a macro-event but with positive probability." (Loewer 2007, 316) A property of decision events that is attractive from Loewer's perspective is that small differences in a decision state can get magnified into large macroscopic differences in the world and he maintains that this feature of "decision conditionals [is] temporally asymmetric": "Alternative decisions that can be made at time $t$ typically can make a big difference to the probabilities of events after $t[\ldots]$ but make no difference to the probabilities of macro events prior to $t . "(317)$

Trying to capture the idea that different decisions are 'open' to an agent at a time, Loewer assumes that decisions are "indeterministic relative to the macro state of the brain and environment prior to, and at the moment of making the decision." (317) From this assumption, it seems, there is an extremely quick argument for the asymmetry of decision counterfactuals. If the assumption is understood not merely as denying determinism but as asserting that decisions are probabilistically independent of the macro-state prior to $t$, it directly follows that differences in decisions "make no difference to the probabilities of macro-events prior to $t$." But this

\footnotetext{
${ }^{10}$ I critically examine several other aspects of Albert's account of the causal asymmetry in (Frisch 2007).
} 
argument for the asymmetry of decision counterfactuals does not rely on the SM account at all and seems question-begging - the asymmetry of decision counterfactuals is simply built into our account of what a decision is. If we want to avoid begging the question, we need to treat Loewer's decision counterfactuals analogous to Albert's causal handles: in evaluating the truth a counterfactuals we hold the actual present macro-state and, in addition, our present memories fixed, posit an alternative decision-event compatible with the state we keep fixed and then let the conditional probabilities of both future and past macro-events be those given by the SM account. For the account to succeed, Loewer's thesis that alternative decisions make no difference to the probabilities of past events would have to come out as a consequence of the SM account.

Yet the SM account fails to imply Loewer's thesis. As a matter of fact our decisions at $t$ are not completely independent of the macro-state of the world prior to $t$-many of my decisions today reflect facts about my biography and are strongly correlated with past experiences. While there may be decisions which amount to mere random 'picking' and hence may be probabilistically independent of my past, many of my decisions exhibit a certain coherence and represent facts both about who I am and about the world. ${ }^{11}$ That is, for many of my decisions there are events $B$ in the past such that $\mathrm{P}(B / D(t)) \neq \mathrm{P}(B /$ not- $D(t))$. Moreover, acknowledging this dependence does not force us to deny Loewer's assumption that different decisions or choices are 'open' to an agent making a decision, since plausibly, this assumption can be captured by supposing that an agent's beliefs and desires do not determine her choices (see Holton 2006, 4) and this supposition is compatible with the claim that an agent's choices are probabilistically correlated with events in her past. Finally, even though my history plays a role in shaping the choices I make, I consciously remember only very few events of my past and only very few of

\footnotetext{
${ }^{11}$ For a discussion of the distinction between 'picking' and 'choosing' see (Holton 2006).
} 
these events have left completely reliable macroscopic traces in the present. Thus, the present macro-state in conjunction with my memories does not screen off the past from my decisionsthat is, for many of my decisions it will be the case that there are past events $B$ such that $\mathrm{P}(B / M(t) \& D(t)) \neq \mathrm{P}(B / M(t)) .^{12}$

Thus, many of an agent's decisions do make a difference to the probabilities of macroevents prior to the time of her making the decision. Now, Loewer argues that even in these cases there still is an important asymmetry between past and future correlations, since, he maintains, we cannot have control over events in our past. According to Loewer, the condition of having control is strictly stronger than the condition of probabilistic dependence: "control by decision requires that there be a probabilistic correlation between the event of deciding that $\mathrm{p}$ be so and $\mathrm{p}$ being so and one's knowing (or believing with reason) that the correlation obtains." (Loewer 2007, 318) Loewer's first condition on control is that there has to be a probabilistic correlation between a decision $D$ and the event $B$ over which have control. This condition, I have argued, is satisfied for large sets of pairs of decisions and events in their past.

A second condition is that we must have good reasons to believe that such a correlation obtains. This condition, too, appears to be frequently satisfied, since we are often in a position to discover how our decisions are correlated with our history. Holton argues that one important role for decision or choice in our lives might be that it enables agents to come to know something about themselves and about the world that they would not have been in a position to know prior to their decision (Holton 2006). According to Holton, by looking at their choices, agents "can

${ }^{12}$ As a putative counterexample to his account Loewer asks whether my decisions now can affect the existence of Atlantis. One implication of my discussion here is that this is not the kind of counterexample about which Loewer should worry. Much more worrisome than the case of Atlantis for Loewer's account are events in the past that the agent facing a decision experienced, but that left (almost) no traces in the present. 
form, rather than just discover, their judgments on that basis" (Holton 2006, 10-11). On

Holton's account, an agent who has the right kinds of competences can in certain circumstances learn from her decisions, since her decisions can act like a finely tuned instrument that picks up on cues that are not consciously available to the agent. If some account like this is correct, then there are correlations between our decisions and past events that we can come to believe with reason and Loewer's second condition is satisfied as well for certain past events.

Yet one might think that while there are many cases where one of the two conditions is satisfied individually, the two conditions can never be jointly satisfied when the events in question lie in the past of a decision. One might think that we can learn of correlations between our decisions and past events only when we remember these events or are in the possession of other reliable records of them, but to the extent that our memories or records are reliable they screen off the past experiences from our present decisions. That is, when the second condition is satisfied, the first condition fails. By contrast, when $\mathrm{P}(B / M(t) \& D(t)) \neq \mathrm{P}(B / M(t))$, we cannot rely on any records to come to know the correlation between our decisions and a past event $B$. That is, when the first condition is satisfied, the second condition fails.

But this objection can be answered. We can learn of correlations between certain kinds of decisions and past events when we do have memories of the past events in question and then use that knowledge inductively to learn something about the past in cases where we make similar decisions but where the relevant memories are absent. This is not much different from how we come to believe reasonably that our decisions are correlated with future events- -by learning inductively from experiences of past correlations.

Here is an example of this. While playing a piano piece that I know well I am unsure whether I am currently playing a part of the piece that is repeated in the score for the first or the 
second time. I decide to play the second ending rather than repeating the part. Many of the notes I play, of course, I play without choosing or deciding to play them. But in the case I am imagining the question what notes to play next has arisen, and I consciously choose to play the second ending. Since I have learned from experience that when I play a piece I know well my decisions to play certain notes are good evidence for where I am in the piece, my present decision not to repeat the part constitutes good evidence for a certain past event-my having already played the part in question once. We can even imagine that I have a vague and unreliable memory of having already played the part. My decision to play the second ending, then can constitute additional evidence for the reliability of my memory. In general, Loewer's first and second conditions are jointly satisfied in cases in which (i) we have good (inductive) reasons for treating our decisions as providing us with information about our past or past events in the world and (ii) the past events in question have left no or only very few and not fully reliable traces in the present.

As a third condition Loewer requires that we have control over an event $B$ only if it is part of the content of our decision that $B$ occur. This third condition is not satisfied for events $B$ in the past of the decision the case of past events, since we do not (normally) take ourselves to have control over the past. But I think this last condition is too strong. We take ourselves to have control over events that are consequences of our decision, even when the content of our decision is not that these events occur. For example, I may have the desire to arrive at the office by 9 a.m. and I have good reasons to believe that my arrival time is reliably correlated with the time when I leave my home. Then my decision to leave at a certain time provides me with a means of controlling when I arrive, even though my decision is, say, a decision to leave at 8 a.m. rather than a decision to arrive by 9 a.m. It seems that we can have control that $p$ be so by 
decision, even when our decision is not a decision that $p$. Thus, two of Loewer's conditions on control by decision can be jointly satisfied by past events, while the third condition should be rejected on independent grounds.

\section{Conclusion}

I have argued that, contrary to what Loewer suggests, it is a consequence of the SM account that there are reconvergences and mergings of possible macro-histories, as well as branchings.

Moreover, the thermodynamic asymmetry results in the destruction of records or traces of the past and there are many 'human-scale' macro-events that leave no or only very few traces in their futures. This result presents serious problems both for Albert's and Loewer's accounts of the asymmetry of causal influence and control. If what I argued in this paper is correct and if the SM account allows that small alterations to the present macro-state and changes to our decisions are probabilistically correlated with changes both to the macro-future and to the macro-past, then Albert and Loewer still owe us an account of how we came to posses strictly time-asymmetric concepts of causal influence or control. 


\section{References}

Albert, David Z. 2000. Time and Chance. Cambridge, Mass.: Harvard University Press.

Baez, John. 2007. The End of the Universe 2004 [cited February 1st 2007]. Available from Causation in Physics/Baez_universe_end.html.

Earman, John. 2006. The "Past Hypothesis": Not even false. Studies in the History and Philosophy of Modern Physics 37 (3):399-430.

Frisch, Mathias. 2000. (Dis-)Solving the Puzzle of the Arrow of Radiation. British Journal for the Philosophy of Science 51:381-410.

-2005. Inconsistency, Asymmetry and Non-locality: A Philosophical Investigation of Classical Electrodynamics. New York: Oxford University Press.

- 2006a. Causal Asymmetry, Counterfactual Decisions and Entropy. Philosophy of Science 72 (5):739-750.

- 2006b. A tale of two arrows. Studies in the History and Philosophy of Modern Physics $37(3): 542-558$.

_ 2007. Causation, Counterfactuals and the Past-Hypothesis. In Russell's Republic: The Place of Causation in the Constitution of Reality, edited by H. Price and R. Corry. Oxford: Oxford University Press.

Holton, Richard. 2006. The Act of Choice. Philosophers' Imprint 6 (3).

Loewer, Barry. 2007. Counterfactuals and the Second Law. In Causality, Physics, and the Constitution of Reality: Russell's Republic Revisited, edited by H. Price and R. Corry. Oxford: Oxford University Press. 
Uffink, Jos. 2006. Compendium to the Foundations of Classical Statistical Physics. In Philosophy of Physics (Handbooks of the Philosophy of Science), edited by J. Butterfield and J. Earman: Elsevier-North Holland.

Winsberg, Eric. 2004. Can Conditioning on the "Past Hypothesis" Militate Against the Reversibility Objection? Philosophy of Science 71:489-504. 
Figure 1

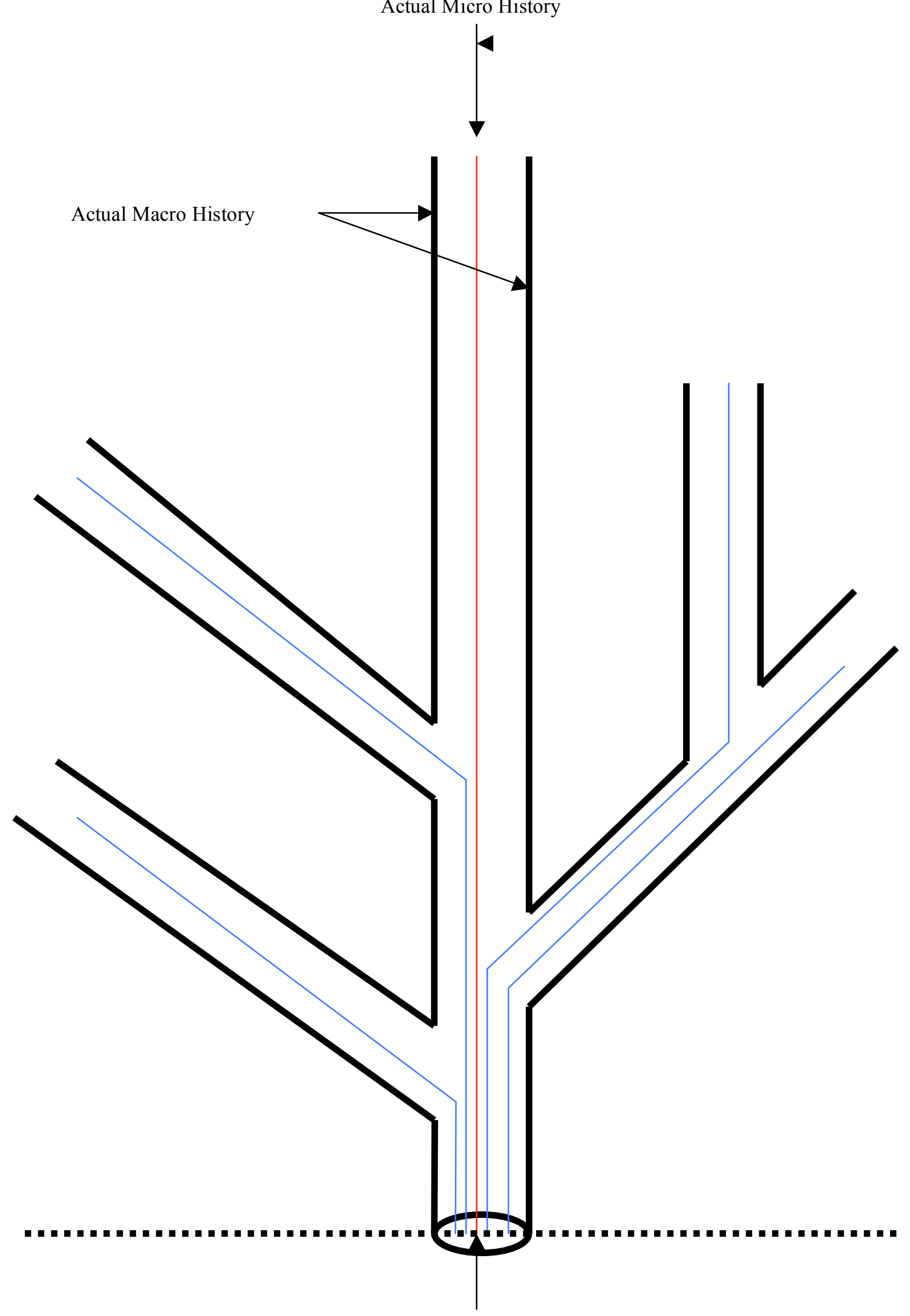

Past Hypothesis

Possible initial conditions 